\title{
A Robust Wearable Textile SIW RFID Antenna
}

\author{
G. A. Casula \\ Dipartimento di Ingegneria Elettrica ed \\ Elettronica \\ Università degli Studi di Cagliari \\ Cagliari, 09123 Italy \\ a.casula@diee.unica.it \\ H. Rogier \\ IDLab-EM group \\ imec-Ghent University \\ B-9052 Gent, Belgium \\ Hendrik.Rogier@ugent.be
}

\author{
G. Montisci \\ Dipartimento di Ingegneria Elettrica ed \\ Elettronica \\ Università degli Studi di Cagliari \\ Cagliari, 09123 Italy \\ giorgio.montisci@unica.it
}

\author{
G. Muntoni \\ Dipartimento di Ingegneria Elettricaed \\ Elettronica \\ Università degli Studi di Cagliari \\ Cagliari, 09123 Italy \\ giacomo.muntoni@diee.unica.it
}

\begin{abstract}
A wearable textile Radio Frequency Identification (RFID) tag realized on a substrate integrated waveguide cavity is presented, operating in the European UHF RFID frequency band. The antenna shows a very good isolation with respect to the human body, and a satisfactory robustness to antenna-body distance variations. The resulting tag is very compact, since its size has been reduced by exploiting the symmetry of a SIW cylindrical resonant cavity and obtaining an eighth mode SIW antenna, and can be produced at low-cost through simple manufacturing processes.
\end{abstract}

Keywords-component, formatting, style, styling, insert (key words)

\section{INTRODUCTION}

Mechanical robustness, easy manufacturing, low-cost, reduced size, light weight, flexibility, reliability in the proximity of the human body, and a low Specific Absorption Rate (SAR) are the main features that distinguish antennas for wearable devices from "conventional" antennas and that allow them to be efficiently integrated into agarment.

The main features of passive RFID tags (low cost, long life, no maintenance, no recharging) make them particularly appropriate for modern applications, such as logistics, retail, security, sensor networks, personal healthcare and so on. A typical RFID device is made of a radio-frequency antenna, connected to a suitable microchip, usually integrated with additional sensors, and is attached to the object to be tagged. In wearable applications, the tag must operate in proximity, or even attached, to the human body, which is a very critical lossy platform. Moreover, the tag antenna size must be kept as small as possible in order to be comfortable for the wearer, and this calls for an accurate design, especially in the UHF RFID frequency bands (from 865 to $970 \mathrm{MHz}$ ).

Many wearable RFID tag antennas proposed in recent literature have been designed using textile substrates [1-5], but their efficiency and gain are strongly degraded by the effects of the human body. Wearable textile antennas based on Substrate Integrated Waveguide (SIW) cavities can improve the isolation from the human body, also allowing a low-profile planar structure, good radiation characteristics, good flexibility [6-8] and multiband operation $[7,8]$.

SIW antennas have been proposed for several applications at $2.4 \mathrm{GHz}$ and beyond, using either coaxial cable or microstrip line feeding [6-8], but SIW technology has not been exploited yet for the design of a RFID passive wearable tag, especially in the lower part of UHF band (around $900 \mathrm{MHz}$ ), where the request of a compact size is still a hard challenge.
In this work, we propose a wearable textile RFID tag antenna based on a substrate integrated waveguide circular cavity, operating within the European UHF frequency band (865-870 MHz). The SIW cavity works at its eight mode (EMSIW), in order to reduce the antenna size, and a further reduction is obtained by optimizing its ground plane size by exploiting the energy-based design strategy proposed in [913], which allow to mitigate the deterioration in antenna performance due to the body proximity.

The antenna design has been performed using CST Microwave Studio, and simulated results show a very good antenna isolation and a satisfying robustness with respect to the antenna-body coupling.

\section{TAG DESIGN AND RESULTS}

The proposed SIW textile wearable tag is designed at a center frequency of $868 \mathrm{MHz}$, and covers the whole frequency European band for RFID Applications (865-870 MHz). The dielectric substrate is composed of a closed-cell rubber foam, typically used in firefighter suits, with a thickness of $4 \mathrm{~mm}$, a dielectric permittivity of 1.3 , and a loss tangent $\tan \delta=0.03$, while the metallic traces have been implemented using a copper coated non-woven PET fabric, with a sheet resistivity of $0.04 \Omega$ /square and a thickness of $0.11 \mathrm{~mm}$. Starting from a SIW cylindrical resonant cavity, operating in the TM010 mode at $868 \mathrm{MHz}$, we exploited the magnetic field symmetry of this $\mathrm{TM}_{010}$ mode to reduce the size of the cavity to obtain the eighth-mode SIW resonator (EMSIW) of Figure 1.

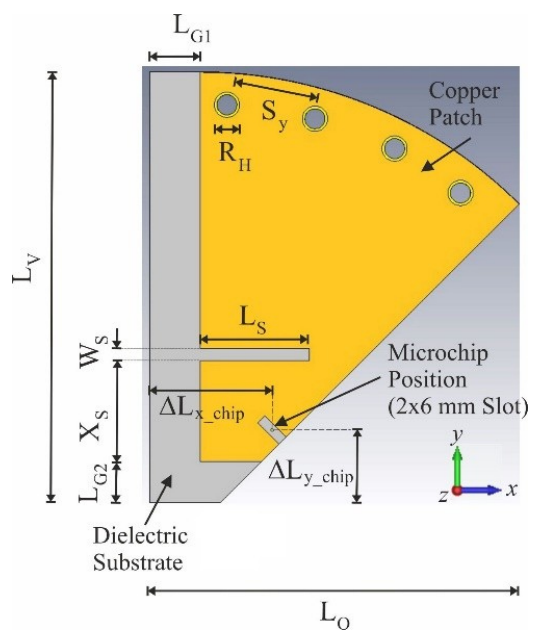

Fig. 1. Layout of the designed SIW antenna. $L_{O}=77.8 \mathrm{~mm}, L_{V}=87.3 \mathrm{~mm}$, $L_{S}=21.7 \mathrm{~mm}, W_{S}=2.5 \mathrm{~mm}, X_{S}=19.9 \mathrm{~mm}, R_{H}=5 \mathrm{~mm}, S_{y}=16 \mathrm{~mm}, \square L_{x \text { chip }}$ $=24.3 \mathrm{~mm}, \square L_{y_{\text {chip }}}=14.4 \mathrm{~mm}, L_{G 1}=L_{G 2}=10 \mathrm{~mm}$ 
In wearable antennas, the antenna electromagnetic performance is strongly influenced by the proximity of the human body, acting as a lossy, non-homogeneous material. Therefore, a very important requirement for a wearable antenna is its robustness. Since the distance between body and antenna randomly changes during antenna operation, the antenna specifications should be satisfied both for deployment in free space and on the human body. A numerical phantom has been added to the simulation scenario (Fig. 2), in order to analyze the body-antenna coupling, and to verify that the antenna performance is still acceptable in close proximity to the human body. We have chosen a simplified phantom, consisting of a single layer with muscle-like parameters at 868 $\mathrm{MHz}\left(\varepsilon_{\mathrm{r}}=56.6, \sigma=1.33 \mathrm{~S} / \mathrm{m}\right)$, with size $250 \times 200 \times 100 \mathrm{~mm}^{3}$ [13].

The main points of the design procedure, leading to the configuration of Fig.1, are:

i) The choice of the ground plane and dielectric substrate extensions: the antenna robustness with respect to the human body proximity is strongly influenced by the ground plane dimension [9-13]. Therefore, an appropriate ground plane extension of both ground plane and dielectric substrate (see Fig. 1) should be kept in proximity of each virtual magnetic wall to achieve a better antenna behavior. In our case, in order to obtain an adequate robustness and isolation for the designed antenna, only the lateral and bottom sides of the ground plane and the substrate have been enlarged, with extensions $\mathrm{L}_{\mathrm{G} 1}=10 \mathrm{~mm}$ and $\mathrm{L}_{\mathrm{G} 2}=10 \mathrm{~mm}$, respectively (see Fig.1).

ii) The addition of a slot of length Ls: cut off in the top patch, as shown in Fig. 1, the slot allows to lengthen the path of the current, further reducing the antenna size, since an increase of Ls reduces the operating frequency. On the other hand, the slot offset $\mathrm{X}_{\mathrm{S}}$ can be used for fine frequency tuning.

iii) The selection of a proper feeding point for the connection of the microchip: the microchip has been placed along the diagonal side of the SIW cavity, as indicated in Fig. 1. This is the most appropriate location, since it minimizes the perturbation of the electromagnetic field inside the cavity. In particular, the position of the microchip has been optimized to easily match the antenna to the input impedance of the Impinj Monza 4 microchip (with a $Z_{\text {chip }}$ equal to 13-j151 $\Omega @$ $868 \mathrm{MHz}$ ).

The design has been performed using CST Microwave Studio through an optimization procedure with the antenna attached to the body phantom ( $\mathrm{d}=0$ in Fig. 3$)$.

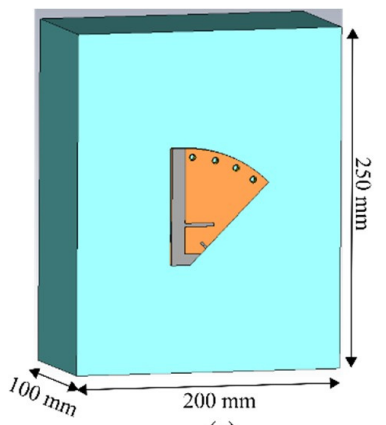

(a)

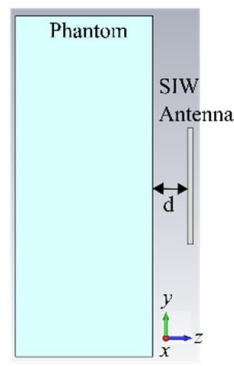

(b)
Fig. 2. Designed antenna with the single layer body phantom: a) 3D view; b) side view.
To assess the antenna robustness according to the energybased design consideration of [9-13], in Fig. 3 the electric energy density distributions in the antenna substrate for $\mathrm{d}=0$ (antenna attached to the body) and $\mathrm{d}=30 \mathrm{~mm}$ are reported at $868 \mathrm{MHz}$. The energy distributions are quite similar, confirming the low sensitivity of the designed antenna with respect to the human body proximity.

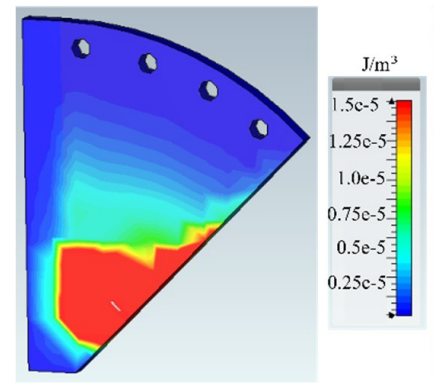

(a)

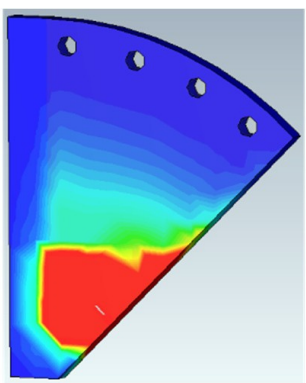

(b)
Fig. 3. Simulated Electric energy density distribution in the antenna substrate. (a) Tag attached to the body $(\mathrm{d}=0)$; (b) tag at $\mathrm{d}=30 \mathrm{~mm}$ from the body.

In Fig. 4, the frequency response of the simulated power transmission coefficient $\tau$ is shown for different spacings $d$ between the antenna and the body phantom. The transmission coefficient is quite stable with respect to different antennabody separations, and the antenna bandwidth is quite large: $\tau$ $>0.8$ in the range $850-890 \mathrm{MHz}$ for $\mathrm{d}=0$, and in the range $859-885 \mathrm{MHz}$ for $\mathrm{d}=30 \mathrm{~mm}$. The proposed structure provides high robustness and isolation with respect to human body coupling, which is a feature depending on the proposed SIW architecture, and is confirmed by the results reported in Figs. 3 and 4.

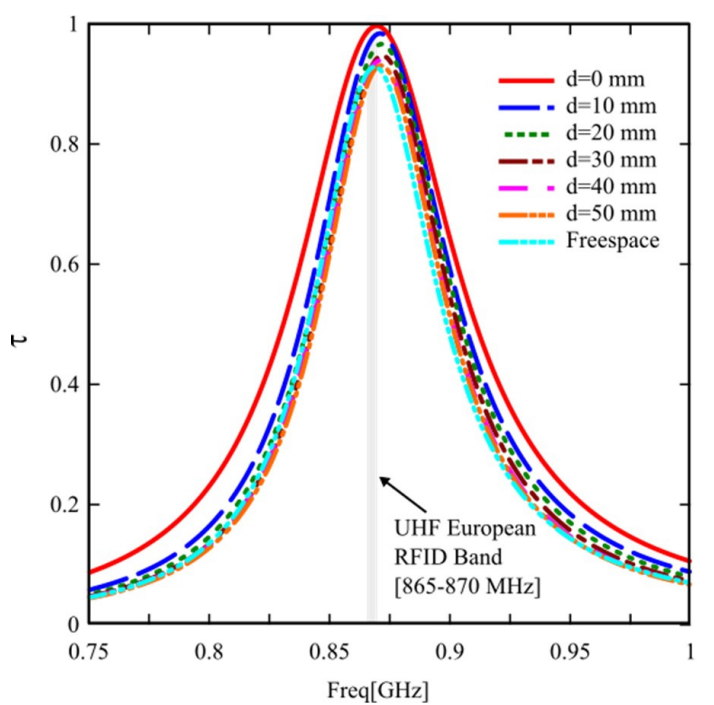

Fig. 4. Simulated power transmission coefficient $\tau$ for different spacings of the antenna from the body phantom.

\section{CONCLUSION}

A wearable textile Radio Frequency Identification (RFID) tag, operating in the European UHF RFID frequency band, has been designed exploiting the SIW technology. Since miniaturization is a key challenge at these frequencies, we choose an Eighth-Mode SIW cavity structure for the tag 
antenna, which allows to achieve a very compact size. The antenna shows a very good isolation with respect to the human body, and a satisfactory robustness to antenna-body distance variations, and can be produced at low-cost through simple manufacturing processes.

\section{REFERENCES}

[1] 1. T. Björninen, "Comparison of three body models of differen complexities in modelling of equal-sized dipole and folded dipole wearable passive UHF RFID tags,” Appl. Comput. Electromagn. Soc. J.1, vol. 33, no. 6, pp. 706-709, June 2018 .

[2] 2. B. Waris, L. Ukkonen, J. Virkki, T. Bjorninen, "Wearable passive UHF RFID tag based on a split ring antenna," IEEE Radio and Wireless Symposium, RWS, art. no. 7885944, pp. 55-58, 2017.

[3] 3. J. Virkki, Z. Wei, A. Liu, L. Ukkonen, T. Björninen, "Wearable passive E-textile UHF RFID tag based on a slotted patch antenna with sewn ground and microchip interconnections," International Journal of Antennas and Propagation, vol. 2017, no. 3476017, Feb. 2017.

[4] 4. T. Kellomäki, "On-body performance of a wearable single-layer RFID tag," IEEE Antennas Wireless Propag. Lett., vol. 11, pp. 73-76, Jan. 2012.

[5] 5. S. Manzari, C. Occhiuzzi, G. Marrocco, "Reading range of wearable textile RFID tags in real configurations", in Proceedings of the 5th European Conference on Antennas and Propagation (EUCAP), Rome, Italy, May 2011.

[6] 6. R. Moro, S. Agneessens, H. Rogier, and M. Bozzi, "Wearable textile antenna in substrate integrated waveguide technology," Electron. Lett., vol. 48, no. 16, pp. 985-987, Aug. 2012.
[7] 7. S. Lemey, F. Declercq, and H. Rogier, "Dual-Band substrate integrated waveguide textile antenna with integrated solar harvester," IEEE Antennas Wireless Propag. Lett., vol. 13, pp. 269-272, Jan. 2014.

[8] 8. S. Agneessens and H. Rogier, "Compact half diamond dual-band textile HMSIW on-body antenna," IEEE Trans. Antennas Propag., vol. 62, no. 5, pp. 2374-2381, May 2014

[9] 9. G.A. Casula, A. Michel, P. Nepa and G. Montisci, "Robustness of Wearable UHF-Band PIFAs to Human-Body Proximity," IEEE Trans. Antennas Propag., vol. 64, no. 5, pp. 2050-2055, May 2016.

[10] 10. A. Michel, R. Colella, G. A. Casula, P. Nepa, L. Catarinucci, G. Montisci, G. Mazzarella, G. Manara, "Design Considerations on the Placement of a Wearable UHF-RFID PIFA on a Compact Ground Plane," IEEE Trans. Antennas Propag., vol. 66, no. 6, pp. 3142-3147, Jun. 2018.

[11] 11. G.A. Casula, G. Montisci, G. Valente, G. Gatto, "A robust printed antenna for UHF wearable applications," IEEE Trans. Antennas Propag., vol. 66, no. 8, pp. 4337-4342, May 2018.

[12] 12. G.A. Casula, G. Montisci, "A Design Rule to Reduce the Human Body Effect on Wearable PIFA Antennas," Electronics, vol. 8, no. 2, Feb. 2019.

13. G. A. Casula, A. Michel, G. Montisci, P. Nepa, and G. Valente, "Energybased considerations for ungrounded wearable UHF antenna design," IEEE Sensors J., vol. 17, no. 3, pp. 687-694, Jan. 2017. 